Article

\title{
Solvent-Free Synthesis of All Silica Beta Zeolite in the Presence of Tetraethylammonium Bromide
}

\author{
Yucun Cui, Zheng Yan, Mengli Li, Jie Zhu, Longfeng Zhu * (1), Hong Yao and Xuebo Cao * \\ College of Biological, Chemical Sciences and Engineering, Jiaxing University, Jiaxing 314001, China; \\ xiaocuigo@163.com (Y.C.); yzheng158@163.com (Z.Y.); uncle.123@163.com (M.L.); zhuyaojie1113@163.com (J.Z.); \\ hongyaoyh@163.com (H.Y.) \\ * Correspondence: zhulf1988@mail.zjxu.edu.cn (L.Z.); xbcao@mail.zjxu.edu.cn (X.C.); \\ Tel.: +86-573-8364-0131 (L.Z. \& X.C.)
}

Received: 31 December 2017; Accepted: 30 January 2018; Published: 1 February 2018

\begin{abstract}
We report a solvent-free synthesis of all silica Beta zeolite using a cheap organic template of tetraethylammonium bromide (TEABr). The method includes mixing, grinding and heating solid raw material in the absence of water solvent but the presence of zeolite seeds. The absence of water solvent significantly improves the efficiency of synthesis, while the addition of seeds remarkably enhances the crystallization rate. In addition, the use of a cheap organic template of TEABr greatly decreases the synthesis cost. The effects of the reaction compositions, including the molar ratios of $\mathrm{NH}_{4} \mathrm{~F} / \mathrm{SiO}_{2}, \mathrm{TEABr} / \mathrm{SiO}_{2}$, and mass ratios of seeds to the silica, on the synthesis of the pure product were investigated using different temperature. Physicochemical characterizations, including XRD, SEM, TG and $\mathrm{N}_{2}$ sorption, show that the zeolitic product has good crystallinity, uniform crystals, and high surface area.
\end{abstract}

Keywords: zeolite; all silica Beta; solvent-free synthesis; tetraethylammonium bromide; cheap

\section{Introduction}

Zeolites are crystalline aluminosilicates and silicates constructed by the $\mathrm{AlO}_{4}$ or $\mathrm{SiO}_{4}$ tetrahedra with various framework structures [1]. Nowadays, zeolites, such as A, X, Y, ZSM-5, ZSM-22, ZSM-23 and Beta, have been successfully commercialized and widely applied in gas adsorption and separation, ion-exchange, and shape selective catalysis [1]. Beta zeolites are among the first synthetic high silica zeolites with three-dimensional large pores with size of $6.6 \times 6.7 \AA$, which is an important material in diverse acid-catalyzed reactions including FCC processes and a basic catalyst for reactions such as alkylation and transesterification [2,3]. Due to the high cost of synthesis, great efforts have been made to improve the synthesis. $\mathrm{Li}$ et al. synthesized the Beta zeolite $(\mathrm{Si} / \mathrm{Al}=8-100)$ using the TEABr as a cheap organic template [4]; Xiao et al. developed the seed-directed synthesis of Al-rich Beta zeolite $(\mathrm{Si} / \mathrm{Al}=4)$ in the absence of organic templates [5]; Wu et al. showed that the combination of solventless synthesis and organotemplate-free routes was used for synthesizing the Al-rich Beta zeolite $(\mathrm{Si} / \mathrm{Al}=6)$. These methodologies greatly decreased the cost of synthesis of aluminosilicates Beta zeolite [6].

On the other hand, all silica Beta zeolite has recently been widely used as catalytic support and adsorbency because of their high surface area, large pore volume, uniform micropores, high hydrophobicity and excellent thermal and hydrothermal stabilities [7-13]. Up to now, all silica Beta zeolite is still hydrothermally synthesized in the presence of water solvent and costly organic templates [14-16]. For example, Wang et al. reported an all silica Beta zeolite synthesis in the presence of a large number of water as solvent and costly TEAOH (aqueous solution) as organic templates in hydrothermal condition [16].

Notably, the hydrothermal route has obvious disadvantages such as reduction of synthesis efficiency owing to autoclave space occupied by the water solvent, and formation of polluted wastes [17-20]. 
The use of expensive organic templates increases the zeolite cost in the production [19,20]. Therefore, developing novel route to synthesize all silica Beta zeolite in the presence of cheap organic templates is strongly desirable.

Recently, we have reported a solvent-free methodology for synthesis of pure silica zeolites (mainly silicalite-1) from anhydrous starting materials in the presence of $\mathrm{NH}_{4} \mathrm{~F}$ [17]. Synthesis parameters and crystallization mechanism on silicalite- 1 have been fully investigated. In this work, we report a solvent-free synthesis of all silica Beta zeolite in detail in the presence of cheap organic template (tetraethylammonium bromide, $\mathrm{TEABr}$ ). The synthetic processes were carefully investigated, and the product would be characterized by X-ray diffraction (XRD), scanning electron microscopy (SEM), $\mathrm{N}_{2}$ sorption, magic angle spinning nuclear magnetic resonance (MAS NMR), and thermogravimetric analysis (TG).

\section{Materials and Methods}

\subsection{Materials}

Tetraethylammonium bromide (TEABr, 98\%, Aladdin Chemistry Co., Ltd., Shanghai, China), solid silica gel $\left(\mathrm{SiO}_{2}\right.$, Qingdao Haiyang Chemical Reagent Co., Ltd., Qingdao, China), and ammonium fluoride $\left(\mathrm{NH}_{4} \mathrm{~F}, 98 \%\right.$, Aladdin Chemistry Co., Ltd., Shanghai, China) were used without further purification.

\subsection{Synthesis}

In a typical run of the synthesis of pure silica zeolite with a *BEA structure, $1.25 \mathrm{~g}$ of $\mathrm{NH}_{4} \mathrm{~F}, 1.6 \mathrm{~g}$ of $\mathrm{SiO}_{2}, 1.75 \mathrm{~g} \mathrm{TEABr}$, and $0.16 \mathrm{~g}$ of pure silica Beta seeds were added into a mortar one by one and mixed together. After grinding for $10 \mathrm{~min}$, the powder was transferred to $15 \mathrm{~mL}$ of an autoclave and sealed. After heating at $140{ }^{\circ} \mathrm{C}$ for $60 \mathrm{~h}$, the sample was fully crystalline. After stirring with $200 \mathrm{~mL}$ water at room temperature for $5 \mathrm{~min}$, then filtrating and drying, the as-synthesized product was finally obtained, giving the weight of $1.45 \mathrm{~g}$. This sample was designated as S-Beta. The $\mathrm{H}$-form of the sample was prepared by calcination at $550{ }^{\circ} \mathrm{C}$ for $6 \mathrm{~h}$. The final product was designated as H-S-Beta.

\subsection{Characterization}

The X-ray diffraction (XRD) patterns were measured with a Rigaku Ultimate VI X-ray diffractometer $(40 \mathrm{kV}, 40 \mathrm{~mA})$ using $\mathrm{CuK} \alpha(\lambda=1.5406 \AA)$ radiation. Scanning electron microscopy (SEM) experiments were performed on Hitachi SU-1510 electron microscopes. The $\mathrm{N}_{2}$ sorption isotherms at the temperature of liquid nitrogen $(77 \mathrm{~K})$ were measured using a Micromeritics ASAP $2020 \mathrm{M}$ and the Tristar system. The ${ }^{29} \mathrm{Si},{ }^{13} \mathrm{C}$, and ${ }^{19} \mathrm{~F}$ solid magic angle spinning nuclear magnetic resonance (MAS NMR) spectra were recorded on a Bruker AVANCE ${ }^{\mathrm{III}} 500 \mathrm{WB}$ spectrometer. Thermogravimetric analysis (TG) was carried out with a NETZSCH STA $449 \mathrm{C}$ in air at a heating rate of $10^{\circ} \mathrm{C} / \mathrm{min}$ from room temperature to $800^{\circ} \mathrm{C}$.

\section{Results and Discussion}

\subsection{Synthesis of S-Beta Zeolite}

Figure 1 shows the XRD pattern of as-synthesized S-Beta zeolite sample in the presence of the TEABr under static conditions for $60 \mathrm{~h}$ at $140^{\circ} \mathrm{C}$, yielding a series of characteristic peaks associated with the ${ }^{*} \mathrm{BEA}$ structure ( $60 \%$ of polymorphs $\mathrm{B}$ and $40 \%$ of polymorphs A) [8]. The low magnification SEM image (Figure 2a) of as-synthesized S-Beta sample shows very uniform crystal morphology, suggesting the high crystallinity of the product obtained. The high magnification SEM image (Figure 2b) of as-synthesized S-Beta zeolite sample exhibits the morphology of truncated octahedral. TG of the as-synthesized S-Beta zeolite sample displays major exothermic peaks at $200-800{ }^{\circ} \mathrm{C}$ accompanied by a weight loss at about $20.04 \%$, which is related to the decomposition of organic template molecules in 
the framework (Figure 3). After calcination at $550{ }^{\circ} \mathrm{C}$ for $6 \mathrm{~h}$, the calcined S-Beta zeolite shows a typical Langmuir adsorption curve (Figure 4), which is due to the filling of nitrogen in the micropores of the S-Beta zeolite sample. Accordingly, the micropore surface area and micropore volume are estimated to be $510 \mathrm{~m}^{2} / \mathrm{g}$ and $0.23 \mathrm{~cm}^{3} / \mathrm{g}$, respectively, which are the same as those of the corresponding Beta zeolite synthesized in the presence of the TEAOH under hydrothermal conditions [8].

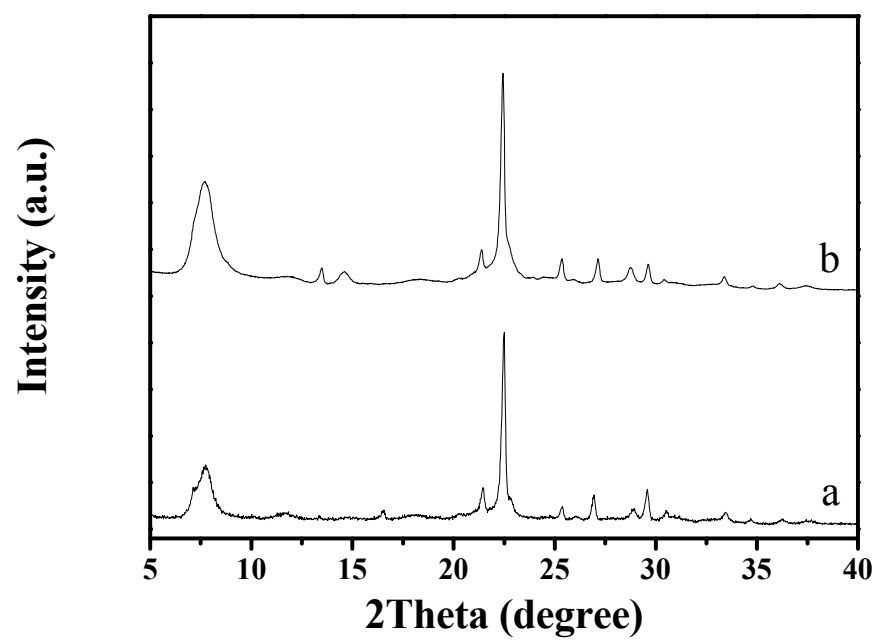

Figure 1. XRD patterns of (a) as-synthesized and (b) calcined S-Beta zeolite.

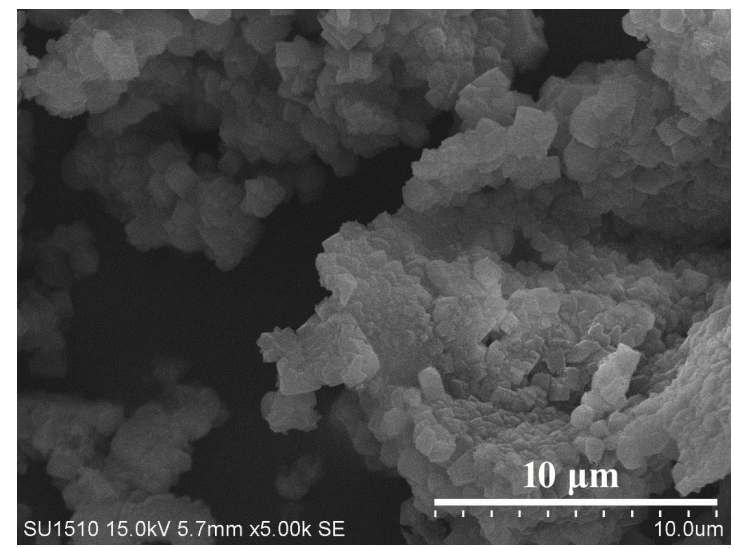

(a)

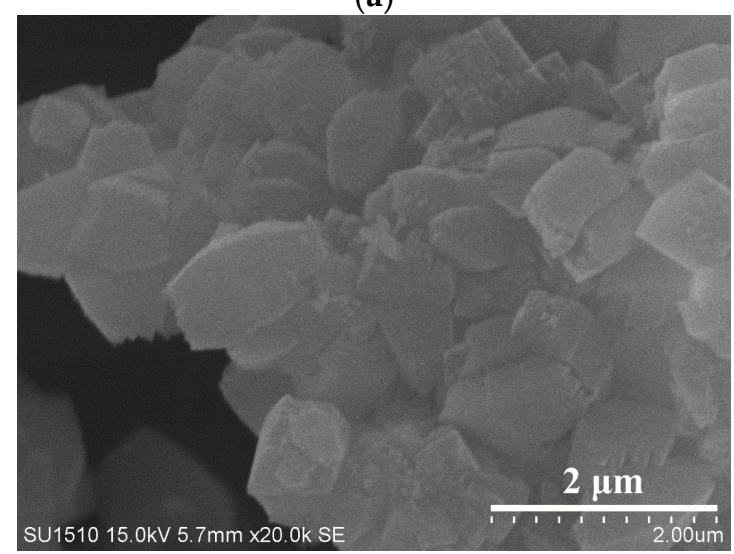

(b)

Figure 2. (a) Low magnification and (b) high magnification SEM images of as-synthesized S-Beta zeolite at $140{ }^{\circ} \mathrm{C}$ for $60 \mathrm{~h}$. 


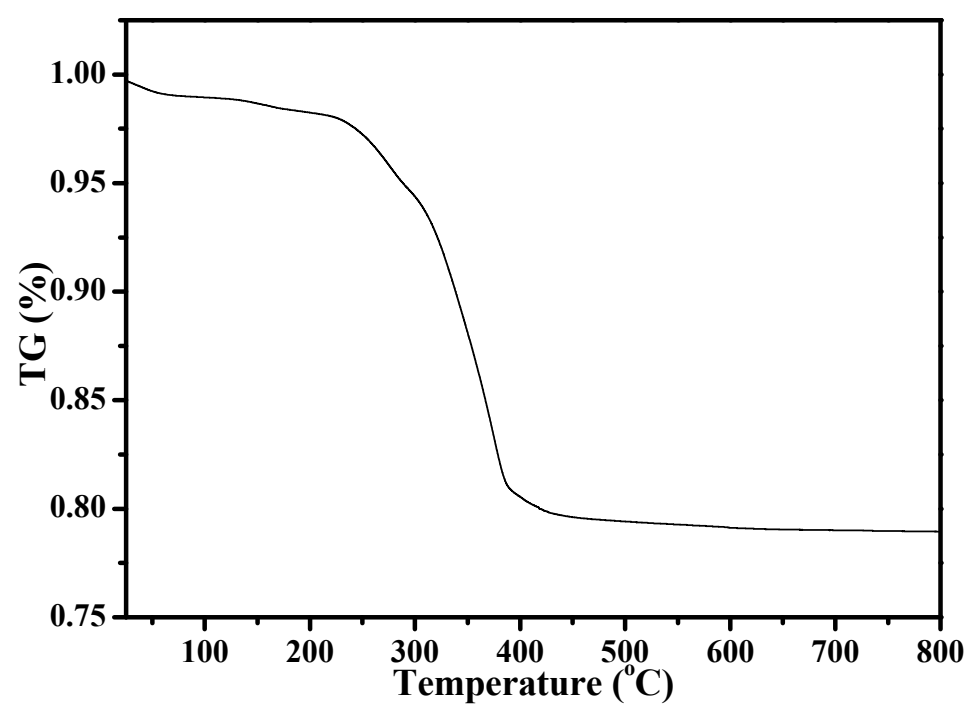

Figure 3. Thermogravimetric analysis (TG) curve of as-synthesized S-Beta zeolite.

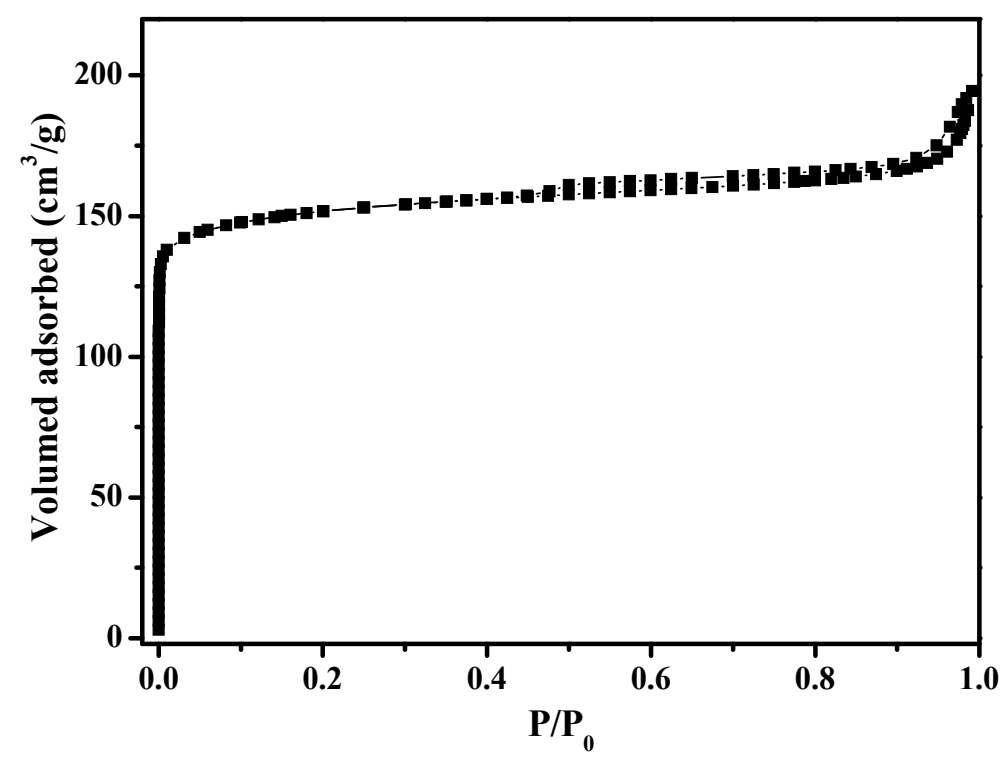

Figure 4. $\mathrm{N}_{2}$ sorption isotherms of calcined S-Beta sample.

Figure 5 shows ${ }^{13} \mathrm{C},{ }^{19} \mathrm{~F}$, and ${ }^{29} \mathrm{Si}$ MAS NMR spectra of as-synthesized S-Beta zeolite sample. Figure 5 a gives the peaks at about 7 and 53 ppm, which are assigned to methyl and methylene in TEA ${ }^{+}$ cation [21]. The ${ }^{29}$ Si MAS NMR spectrum (Figure 5b) exhibits five bands at about $-116.2,-113.3$, $-110.5,-108.4$, and $-104.6 \mathrm{ppm}$. These bands are assigned to $\mathrm{Si}(4 \mathrm{Si})$ species [18]. The ${ }^{19} \mathrm{~F}$ MAS NMR spectrum (Figure 5c) shows the three peaks at $-37.7,-57.5$, and $-69.6 \mathrm{ppm}$. The first signal at $-37.7 \mathrm{ppm}$ is due to the fluoride ion occluded in the D4R cage, whereas the latter two signals are attributed to the fluoride ions occluded in the $\left[4^{3} 5^{4}\right]$ cage of S-Beta zeolite sample. Due to be short of D4R cage in S-Beta zeolite sample, the detected D4R in the sample is most likely associated with the existence of the two adjacent layers at least stacked with the manner in polymorph $C$, which is in good agreement with the literature [15]. 

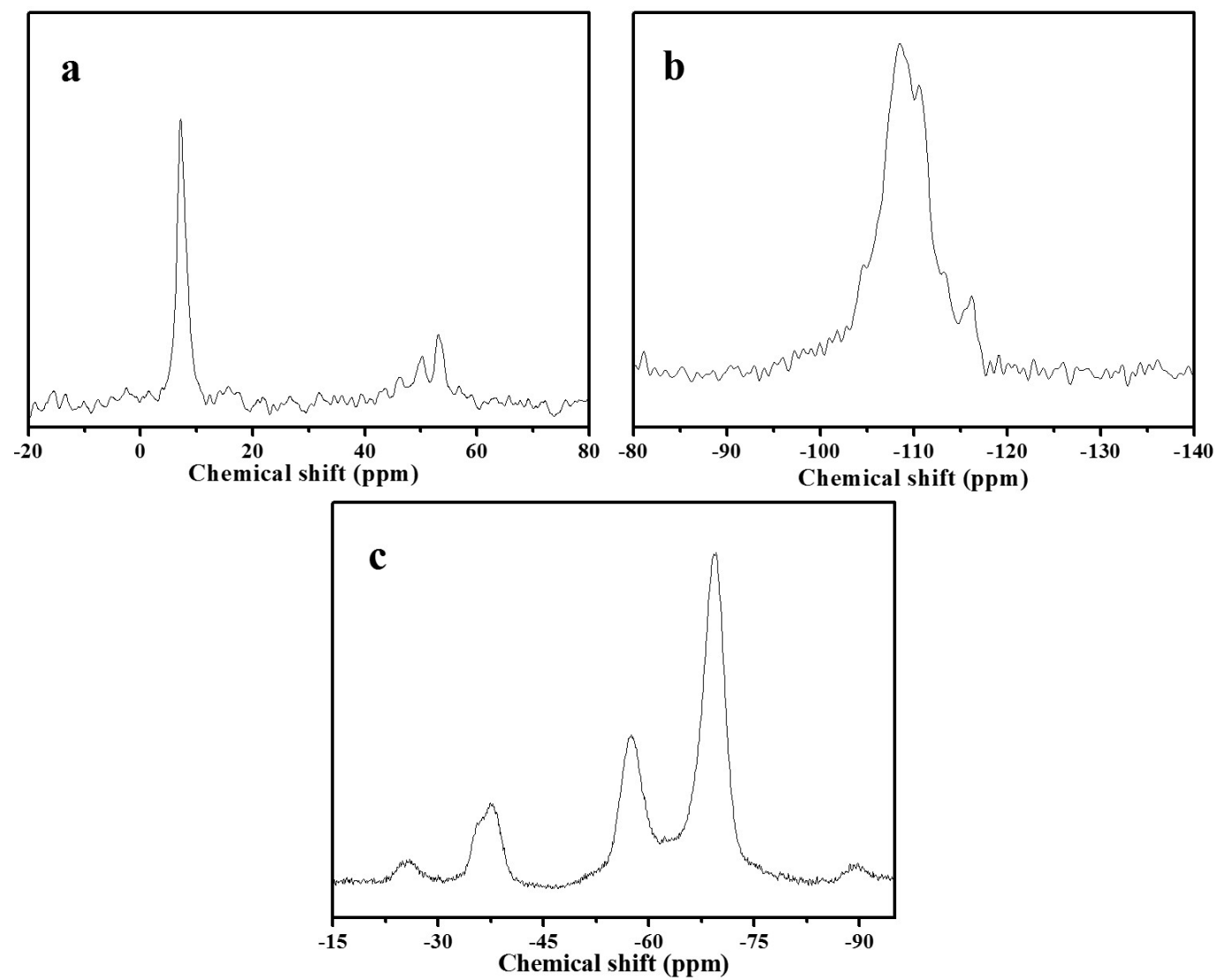

Figure 5. (a) ${ }^{13} \mathrm{C}$; (b) ${ }^{29} \mathrm{Si}$ and (c) ${ }^{19} \mathrm{~F}$ MAS NMR spectra of as-synthesized S-Beta sample after water treatment.

\subsection{Critical Factors for Solvent-Free Synthesis of S-Beta Zeolite}

Various factors for solvent-free synthesis of S-Beta zeolite have been carefully investigated, and it was found that the ratios of $\mathrm{NH}_{4} \mathrm{~F} / \mathrm{SiO}_{2}, \mathrm{TEABr} / \mathrm{SiO}_{2}$, synthesis temperature, and addition of seeds in the starting solid mixture were important to S-Beta zeolite synthesis, as shown in Table 1 and Figure 6.

Table 1. Synthesis of S-Beta sample under various conditions.

\begin{tabular}{cccccc}
\hline Run & Seed $(\%)^{\mathbf{b}}$ & $\mathbf{N H}_{\mathbf{4}} \mathbf{F} / \mathbf{S i O}_{\mathbf{2}}$ & $\mathbf{T E A B r} / \mathbf{S i O}_{\mathbf{2}}$ & Temperature $\left({ }^{\circ} \mathbf{C}\right)$ & Products \\
\hline 1 & 0 & 1.25 & 0.3 & 140 & Amor + Beta \\
$2^{\mathrm{a}}$ & 10 & 1.25 & 0.3 & 140 & Beta \\
3 & 10 & 0.5 & 0.3 & 140 & Amor + Beta \\
4 & 10 & 1.0 & 0.3 & 140 & Beta + Amor \\
5 & 10 & 1.25 & 0.15 & 140 & Amor + Beta \\
6 & 10 & 1.25 & 0.25 & 140 & Beta + Amor \\
7 & 10 & 1.25 & 0.3 & 160 & MFI + Beta \\
8 & 10 & 1.25 & 0.3 & 120 & Beta + Amor \\
\hline
\end{tabular}

a Characterizations are shown in Figures $1-7 ;^{\mathrm{b}}$ Mass ratios of seeds to the silica source; ${ }^{\mathrm{c}}$ The phase appearing first is dominant. 


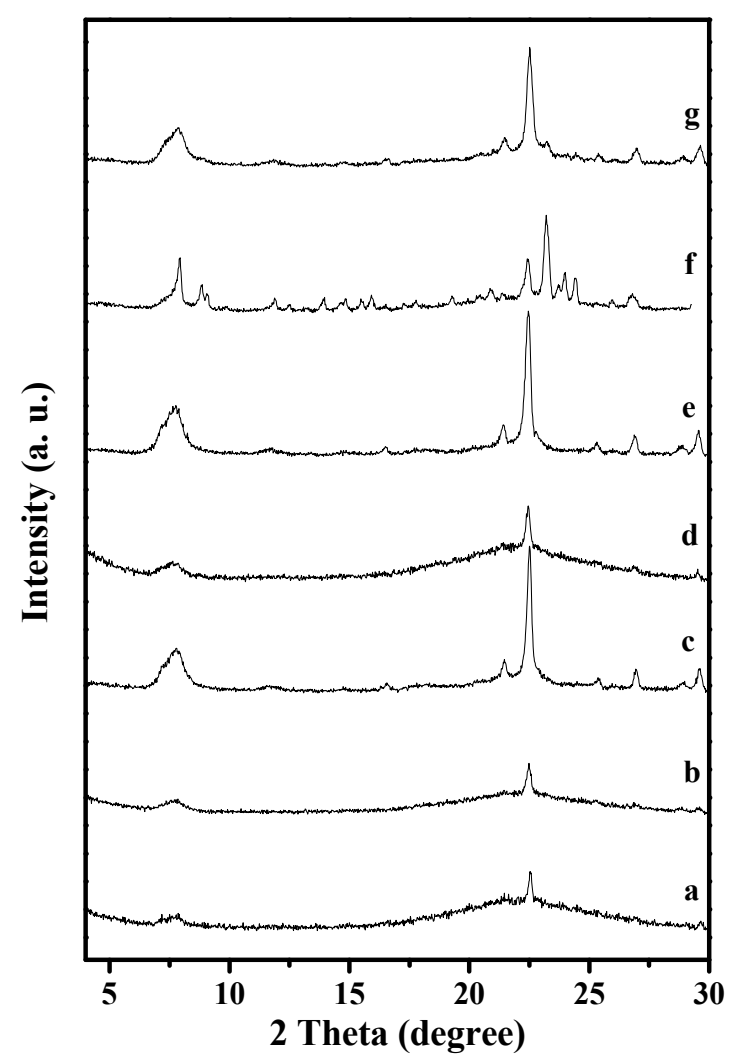

Figure 6. XRD patterns of solid samples synthesized from the starting gels with molar ratio of 1.0 $\mathrm{SiO}_{2} / 0.5-1.25 \mathrm{NH}_{4} \mathrm{~F} / 0.15-0.3 \mathrm{TEABr}$ with mass ratios of $0-10 \%$ seeds to the silica source at $120-160{ }^{\circ} \mathrm{C}$ for $60 \mathrm{~h}$. (a) $0 \%$ Seeds to the silica source; $\mathrm{NH}_{4} \mathrm{~F} / \mathrm{SiO}_{2}$ ratios of (b) 0.5 ; and (c) 1.0; $\mathrm{TEABr} / \mathrm{SiO}_{2}$ ratios of (d) 0.15 ; and (e) 0.25 ; (f) $160{ }^{\circ} \mathrm{C}$ for $60 \mathrm{~h}$; (g) $120{ }^{\circ} \mathrm{C}$ for $60 \mathrm{~h}$.

Notably, Beta seeds play an important role in the synthesis. If Beta seeds are absent in the solid starting mixtures, a majority of products are amorphous (Run 1 in Table 1 and Figure 6a). After addition of Beta seeds to the starting mixtures, S-Beta zeolites with good crystallinity are obtained (Run 2 in Table 1 and Figure 1). In addition, the addition of seeds remarkably enhances the crystallization rate, with $60 \mathrm{~h}$ at $140{ }^{\circ} \mathrm{C}$, compared with traditional hydrothermal synthesis $\left(168 \mathrm{~h}\right.$ at $\left.140{ }^{\circ} \mathrm{C}\right)$ [13].

The amount of $\mathrm{NH}_{4} \mathrm{~F}$ is a key factor for S-Beta synthesis. Too little $\mathrm{NH}_{4} \mathrm{~F}$ results in amorphous phase in the final product (Run 3 and 4 in Table 1 and Figure $6 \mathrm{~b}, \mathrm{c}$ ). When the $\mathrm{NH}_{4} \mathrm{~F} / \mathrm{SiO}_{2}$ ratio reaches 1.25, pure S-Beta zeolite product with good crystallinity is obtained (Run 2 in Table 1 and Figure 1).

The ratio of $\mathrm{TEABr} / \mathrm{SiO}_{2}$ strongly influences the final products in this synthesis. If this ratio was very low (Run 5 and 6 in Table 1 and Figure 6d,e), amorphous phase always existed in the final samples. When this ratio is 0.3 , pure S-Beta zeolite product with tetragonal $\mathrm{P}_{1} 22$ [21] could be obtained (Run 2 in Table 1 and Figure 1).

Moreover, the crystallization temperature is very important for synthesis of S-Beta zeolite. When the crystallization temperature is $160{ }^{\circ} \mathrm{C}$, MFI zeolite with orthorhombic Pnma [21] is dominant (Run 7 in Table 1 and Figure 6f), and a little amount of all silica Beta zeolite is still formed, which lead to the strong broadness in particular around 2 theta of 7.5 degree; when the crystallization temperature decreases to $140{ }^{\circ} \mathrm{C}$, S-Beta zeolite is obtained (Run 2 in Table 1 and Figure 1); when the crystallization temperature further decreases to $120^{\circ} \mathrm{C}$, the product obtained contains amorphous phase (Run 8 in Table 1 and Figure 6g).

Based on these results (Table 1 and Figure 6), it could be concluded that the crystallization of S-Beta zeolite is strongly influenced by many factors and the pure phase of S-Beta zeolite could only 
be obtained in a narrow phase diagram of $\mathrm{NH}_{4} \mathrm{~F}-\mathrm{TEABr}-\mathrm{SiO}_{2}$-Seed at appropriate temperature by a comprehensive consideration of these factors.

\subsection{Crystallization Process of S-Beta Zeolite}

To understand the solvent-free synthesis of S-Beta zeolite in the presence of the cheap TEABr, the crystallization process at $140{ }^{\circ} \mathrm{C}$ was characterized by XRD and SEM techniques (Figures 7 and 8). The dependence of sample crystallinity on crystallization time for S-Beta zeolite sample is shown in Figure 9. Before crystallization, the sample shows very weak peaks associated with the *BEA structure (Figure 7a), which are related to the Beta seeds existing in the starting mixtures. After crystallization for $6 \mathrm{~h}$, the sample displays stronger peaks associated with Beta zeolite (Figure 7c) though the amorphous phase is still dominant. After increasing the time from $12-48 \mathrm{~h}$, the peak intensity related to Beta zeolite significantly increases (Figure 7d-h). At the same time, SEM images of the samples shows that more truncated octahedral analogous crystals can be observed (Figure 8c-e). When the crystallization time reach $60 \mathrm{~h}$, the Beta zeolite with high crystallinity and uniform crystals are obtained ( Figures 7i and 8f). Further increasing the crystallization time to $72 \mathrm{~h}$, the intensity of XRD peaks does not change, which indicates the crystallization is completely finished (Figure 7j). These results confirm the rapid synthesis of S-Beta zeolite in the presence of Beta seed under solvent-free condition.

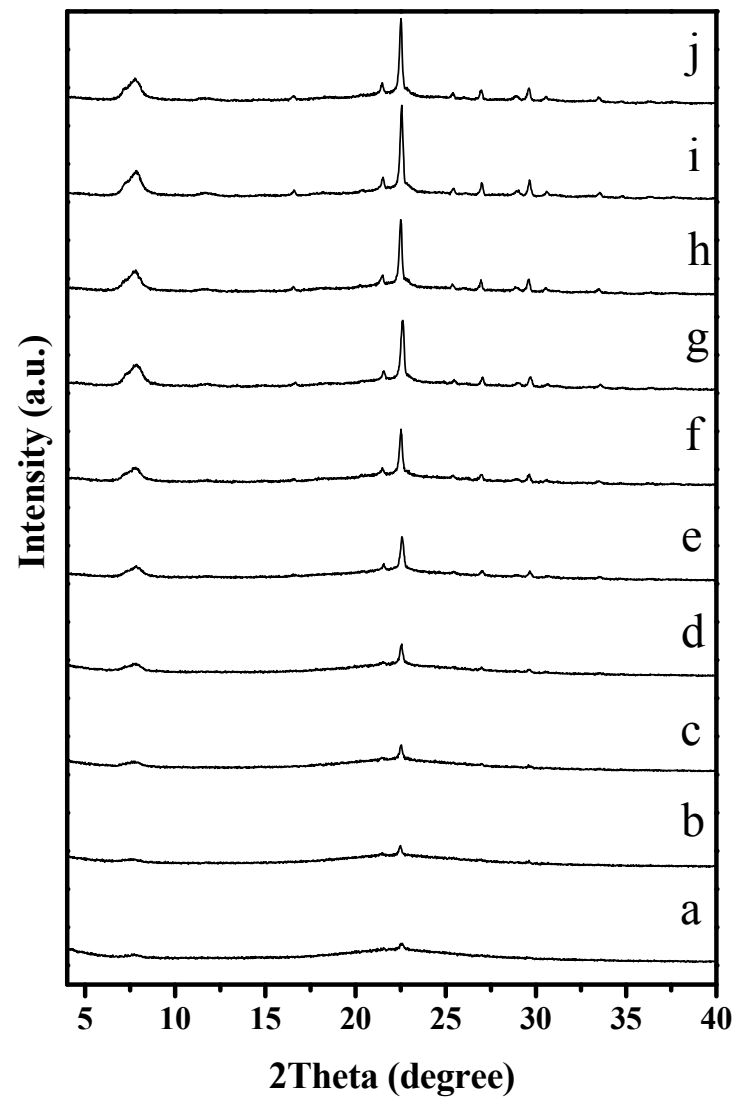

Figure 7. XRD patterns of S-Beta samples with tetragonal $\mathrm{P}_{1} 22$ crystallized for (a) 0 ; (b) 3; (c) 6; (d) 12; (e) $18 ;$ (f) $24 ;$ (g) 36 ; (h) 48; (i) 60 and (j) 72 h, respectively. 


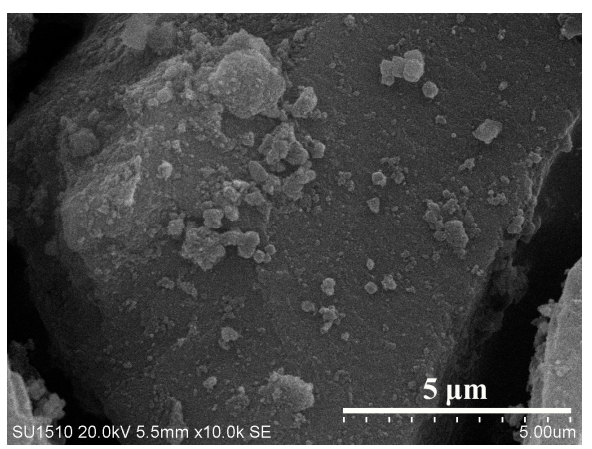

(a)

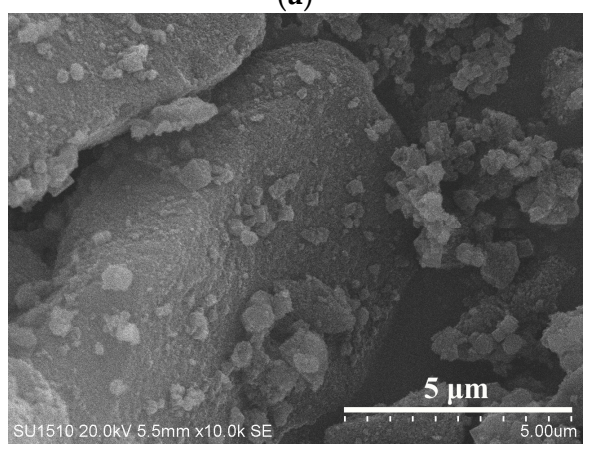

(c)

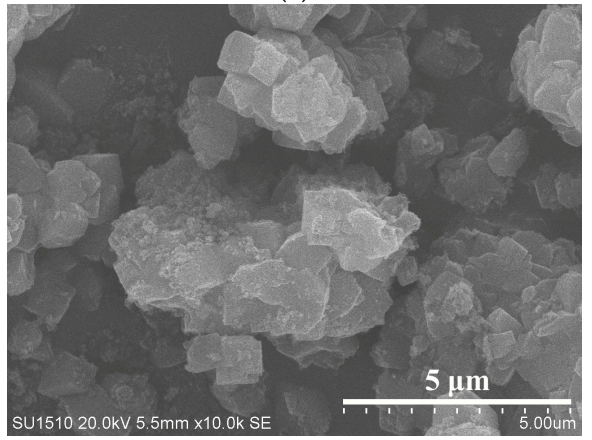

(e)

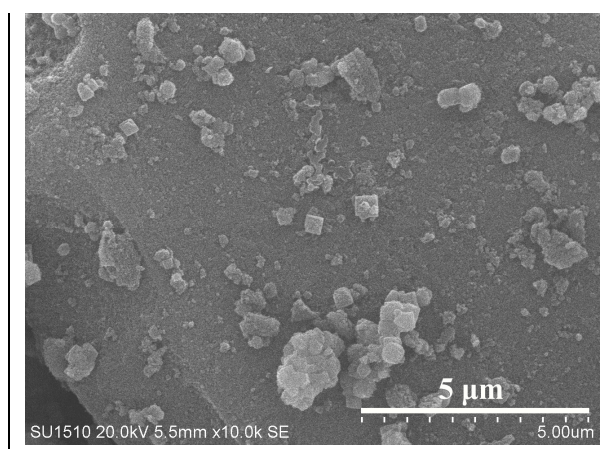

(b)

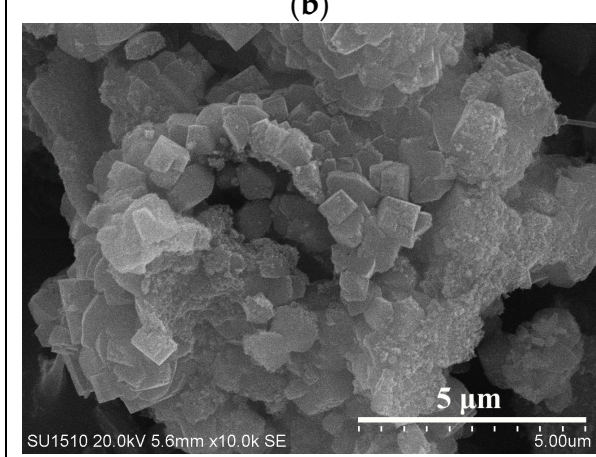

(d)

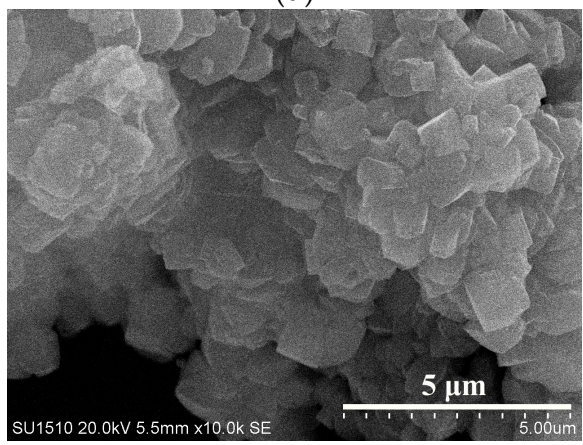

(f)

Figure 8. SEM images of S-Beta samples crystallized for (a) 0; (b) 6; (c) 18; (d) 36; (e) 48 and (f) $60 \mathrm{~h}$, respectively.

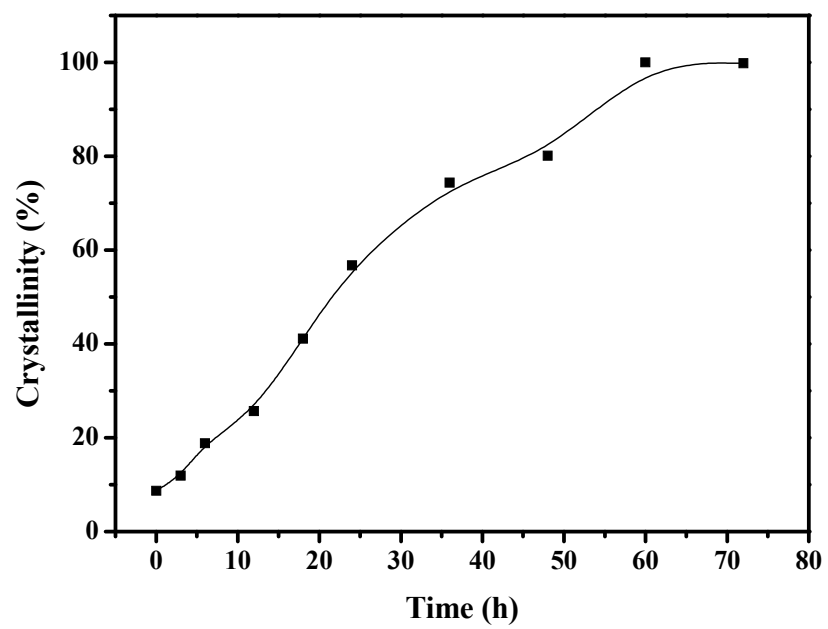

Figure 9. The dependence of S-Beta crystallinity over crystallization time. 


\section{Conclusions}

In summary, we use TEABr as a cheap and effective organic template for successfully synthesizing all silica S-Beta zeolite in the absence of water, but in the presence of zeolite seeds. It is the first time for systematically synthesizing S-Beta zeolite using the TEABr. Compared with traditional hydrothermal synthesis, this synthesis route has obvious advantages from the sustainability point of view with respect to high product yield, cheap organic template, simple procedures, and comparatively short time. These features should be potentially important for industrial application of all silica Beta zeolite as the catalytic support and adsorbent candidate in the near future.

Acknowledgments: This work is supported by the National Training Program of Innovation for Undergraduates (201610354021), the National Nature Science Foundation of China (21501067), and the Natural Science Foundation of Zhejiang Province (LY15B030008). We thank Yuyin Wan, Jiangtao Zheng and Jie Wang for the contribution of reagents/materials/analysis tools.

Author Contributions: Yucun Cui and Zheng Yan conceived and designed the experiments; Hong Yao performed the experiments; Mengli Li and Jie Zhu analyzed the data; Longfeng Zhu and Xuebo Cao wrote the paper.

Conflicts of Interest: The authors declare no conflict of interest.

\section{References}

1. Davis, M.E.; Lobo, R.F. Zeolite and molecular sieve synthesis. Chem. Mater. 1992, 4, 756-768. [CrossRef]

2. Corma, A.; Gonzalez-Alfaro, V.; Orchilles, A.V. The role of pore topology on the behaviour of FCC zeolite additives. Appl. Catal. A 1999, 187, 245-254. [CrossRef]

3. Meyer, U.; Hoelderich, W.F. Transesterification of methyl benzoate and dimethyl terephthalate with ethylene glycol over basic zeolites. Appl. Catal. A 1999, 178, 159-166. [CrossRef]

4. Niu, T.Y.; Li, Y.X.; Li, H.W.; Chen, B.H. Synthesis of zeolite Beta by the vapour-phase transport method using tetraethylammonium bromide as the organic template. Chem. J. Catal. 2009, 30, 191-195. [CrossRef]

5. Xie, B.; Song, J.; Ren, L.; Ji, Y.; Li, J.; Xiao, F.-S. Organotemplate-free and fast route for synthesizing beta zeolite. Chem. Mater. 2008, 20, 4533-4535. [CrossRef]

6. Wu, Q.M.; Wang, X.; Qi, G.D.; Guo, Q.; Pan, S.X.; Meng, X.J.; Xu, J.; Deng, F.; Fan, F.T.; Feng, Z.C.; et al. Sustainable Synthesis of Zeolites without Addition of Both Organotemplates and Solvents. J. Am. Chem. Soc. 2014, 136, 4019-4025. [CrossRef] [PubMed]

7. Cosseron, A.F.; Daou, T.J.; Tzanis, L.; Nouali, H.; Deroche, I.; Coasne, B.; Tchamber, V. Adsorption of volatile organic compounds in pure silica CHA, BEA, MFI and STT-type zeolites. Microporous Mesoporous Mater. 2013, 173, 147-154. [CrossRef]

8. Zhu, Z.G.; Xu, H.; Jiang, J.G.; Wu, H.H.; Wu, P. Hydrophobic Nanosized All-Silica Beta Zeolite: Efficient Synthesis and Adsorption Application. ACS Appl. Mater. Interfaces 2017, 9, 27273-27283. [CrossRef] [PubMed]

9. Chen, Y.L.; Zhu, G.S.; Peng, Y.; Bi, H.; Feng, J.; Qiu, S.L. Synthesis and characterization of pure-silica-zeolite Beta low-k thin films. Microporous Mesoporous Mater. 2009, 123, 45-49. [CrossRef]

10. Larlus, O.; Mintova, S.; Wilson, S.T.; Willis, R.R.; Abrevaya, H.; Bein, T. A powerful structure-directing agent for the synthesis of nanosized Al-and high-silica zeolite Beta in alkaline medium. Microporous Mesoporous Mater. 2011, 142, 17-25. [CrossRef]

11. Jo, C.; Park, W.; Ryoo, R. Synthesis of mesoporous zeolites in fluoride media with structure directing multiammonium surfactants. Microporous Mesoporous Mater. 2017, 239, 19-27. [CrossRef]

12. Weng, Q.; Chen, P.; Zhao, F.Q.; Gao, H.X.; Chen, X.B.; An, Z.W. Synthesis and Characterization of Hierarchical Aluminum Free Beta Zeolite. Chem. J. Chin. Univ. 2014, 35, 215-223.

13. Kuechl, D.E.; Benin, A.I.; Knight, L.M.; Abrevaya, H.; Wilson, S.T.; Sinkler, W.; Mezza, T.M.; Willis, R.R. Multiple paths to nanocrystalline high silica beta zeolite. Microporous Mesoporous Mater. 2010, 127, 104-118. [CrossRef]

14. Taborda, F.; Willhammar, T.; Wang, Z.Y.; Montes, C.; Zou, X.D. Synthesis and characterization of pure silica zeolite beta obtained by an aging-drying method. Microporous Mesoporous Mater. 2011, 143, 196-205. [CrossRef] 
15. Tong, M.Q.; Zhang, D.L.; Fan, W.B.; Xu, J.; Zhu, L.K.; Guo, W.; Yan, W.F.; Yu, J.H.; Qiu, S.L.; Wang, J.G.; et al. Synthesis of chiral polymorph A-enriched zeolite Beta with an extremely concentrated fluoride route. Sci. Rep. 2015, 5, 11521. [CrossRef] [PubMed]

16. Wang, Y.Y.; Tsai, C.H.; Tsai, C.H. Method for Synthesizing All-Silica Zeolite Beta with Small Crystal Size. U.S. Patent 8,206,683, 26 June 2012.

17. Wu, Q.M.; Liu, X.L.; Zhu, L.F.; Ding, L.H.; Gao, P.; Wang, X.; Pan, S.X.; Bian, C.Q.; Meng, X.J.; Xu, J.; et al. Solvent-Free Synthesis of Zeolites from Anhydrous Starting Raw Solids. J. Am. Chem. Soc. 2015, 137, 1052-1055. [CrossRef] [PubMed]

18. Zhu, L.F.; Zhang, J.; Wang, L.; Wu, Q.M.; Bian, C.Q.; Pan, S.X.; Meng, X.J.; Xiao, F.S. Solvent-free synthesis of titanosilicate zeolites. J. Mater. Chem. A 2015, 3, 14093-14095. [CrossRef]

19. Wu, Q.M.; Liu, X.L.; Zhu, L.F.; Meng, X.J.; Deng, F.; Fan, F.T.; Feng, Z.C.; Li, C.; Maurer, S.; Feyen, M.; et al. Solvent-Free Synthesis of ITQ-12, ITQ-13, and ITQ-17 Zeolites. Chin. J. Chem. 2017, 35, 572-576. [CrossRef]

20. Meng, X.J.; Xiao, F.S. Green Routes for Synthesis of Zeolites. Chem. Rev. 2014, 114, 1521-1543. [CrossRef] [PubMed]

21. Liu, X.L.; Luo, Q. Solid State NMR Spectroscopy Studies of the Nature of Structure Direction of OSDAs in Pure-Silica Zeolites ZSM-5 and Beta. J. Phys. Chem. C 2017, 121, 13211-13217. [CrossRef]

(C) 2018 by the authors. Licensee MDPI, Basel, Switzerland. This article is an open access article distributed under the terms and conditions of the Creative Commons Attribution (CC BY) license (http:/ / creativecommons.org/licenses/by/4.0/). 